\title{
Resuscitation room blood alcohol concentrations: one-year cohort study
}

\author{
R Touquet, ${ }^{1}$ E Csipke, ${ }^{3}$ P Holloway, ${ }^{1}$ A Brown, ${ }^{2}$ T Patel, ${ }^{1}$ A J Seddon, ${ }^{1}$ P Gulati, \\ H Moore, ${ }^{1}$ N Batrick, ${ }^{1}$ M J Crawford ${ }^{3}$
}

${ }^{1}$ Imperial College Healthcare NHS Trust, London, UK; ${ }^{2}$ Central and North West London Foundation Trust, London, UK; ${ }^{3}$ Imperial College London, London, UK

Correspondence to: Professor R Touquet, Accident and Emergency Department, St Mary's Hospital, London W2 1NY, UK; robin.touquet@ imperial.nhs.uk

Accepted 9 July 2008

\begin{abstract}
Objective: To clarify the relationship between presenting clinical condition and blood alcohol concentration (BAC) among adult patients admitted to a resuscitation room (RR) of an emergency department (ED) in order to help guide clinical practice.
\end{abstract}

Method: Single-site prospective cohort study of all patients admitted to the RR of an inner-city hospital over a one-year period. The study sample comprised all those aged 16 years and over from whom a blood sample was taken, with BAC (results not known to ED staff). pathology by International Classification of Diseases (ICD) version 10 coding, injury severity score for trauma, return visit to hospital and mortality during the subsequent 6 month period, being recorded.

Results: 291 (15\%) of 1908 presentations had a positive BAC (ie, BAC $>10 \mathrm{mg} / 100 \mathrm{ml}$ ) ranging from 11 to $574 \mathrm{mg} / 100 \mathrm{ml}$, of which almost 40\% were over $240 \mathrm{mg} / 100 \mathrm{ml}$ (ICD-10 code Y90.8). In addition to collapse from alcohol/drugs, almost half of those presenting following self-harm or assault had a positive $\mathrm{BAC}$. Those with a positive BAC had a higher rate of $\mathrm{ED}$ re-attendance in the following 6 months. 10\% of all presentations were due to trauma.

Conclusion: The following five presentations to the RR are associated with a positive BAC: collapse from alcohol/ drugs, self-harm, trauma, gastrointestinal bleeding (ICD10 code K92.2) and non-cardiac chest pain (ICD-10 code R07). Patients with a positive BAC demonstrate a very wide range of pathology, some with severe levels of misuse. This highlights the opportunity for prompt feedback when sober, to ensure all is done to encourage patients to contemplate change in order to reduce reattendance.

This work follows directly on from our first paper: "Use of blood alcohol concentrations in resuscitation room patients", ${ }^{1}$ which covers ethical, judicial and insurance issues. Alcohol misuse is a very common problem confronting all healthcare staff working in emergency departments (ED) worldwide. ${ }^{2}$ The value of screening and brief intervention in ED by an alcohol nurse specialist is established, ${ }^{3}$ with one less return visit to the ED over 12 months for every two people referred to an alcohol nurse specialist. ${ }^{4}$ At our hospital early identification of alcohol misuse is by the Paddington alcohol test (PAT) facilitating brief advice (with possible referral for brief intervention). ${ }^{5}$

Identification of patients with alcohol problems in the ED may be by history (PAT), clinical examination or blood alcohol concentration (BAC). PAT application is usually not possible for patients who are potentially critically ill and the need is for urgent assessment and treatment. Clinical examination for possible alcohol use is limited for the obtunded patient and has variable concordance with BAC. ${ }^{6}$ A degree of tolerance, thereby camouflaging clinical signs, for the "experienced" drinker is also well recognised." Measurement of BAC may provide a means of assessing the influence of alcohol use on the person's presentation and management, ${ }^{8-10}$ but our ED staff were blinded to results (BAC requests not previously being routinely available at our hospital). Requesting the BAC without prior consent is acceptable to potentially critically ill patients, provided that feedback is given when patients are in a sufficiently improved clinical state. ${ }^{11}$

The extent of concurrent alcohol use among all categories of patients treated specifically in the resuscitation room (RR) of an $E D$ has not previously been reported; every ED in the UK has a specific RR, usually between two and six bays, for potentially critically ill patients. ${ }^{12}$ We therefore set out to determine which RR patients were most likely to have a positive BAC and to explore associations with clinical outcomes, now that early identification with the giving of brief advice has been shown to be effective in reducing $\mathrm{ED}$ reattendance.

\section{METHODS}

The study took place in the four-bayed adult RR of the ED of an acute hospital serving a metropolitan inner-city population of over 500000 adults, seeing 65000 new adult (16 years and over) ED visits each year. We measured BAC over a 12-month period from all blood samples taken from patients initially presenting to the $\mathrm{RR}$, with the exception of direct admissions to specialists and transfers from other hospitals (before this BAC testing was not available on our hospital site). The spectrum of disease of those patients presenting to the RR was broad (see tables 1 and 2). All had life-threatening, or potentially life-threatening pathology, triage categories 1 or $2 .{ }^{12}$ Patients under 16 years of age were excluded. The medical and nursing staff were blinded to BAC results, which therefore had no influence on patient management. The local research ethics committee approved this study as an audit, therefore informed consent was waived (no EC2143 dated 31 March 2005).

Blood samples were collected into a fluorideoxalate bottle (as for blood sugar estimation); BAC was measured by an automated enzymatic method-DRI ethyl alcohol assay (Microgenics online under the BMJ Journals unlocked scheme, see http:// emj.bmj.com/info/unlocked.dtl 
Table 1 Characteristics of 2137 episodes in which BAC was and was not tested

\begin{tabular}{|c|c|c|c|}
\hline & Not tested & Tested & Total \\
\hline & $N=229$ & $N=1908$ & $N=2137$ \\
\hline $\begin{array}{l}\text { Gender (male) } \\
\quad N=2137\end{array}$ & $106(46 \%)$ & $739(39 \%)$ & $845(40 \%)$ \\
\hline $\begin{array}{l}\text { Mean age (SD) } \\
\qquad N=2137\end{array}$ & $56.80(20.52)$ & $55.64(21.04)$ & $56.67(20.58)$ \\
\hline $\begin{array}{l}\text { Ethnicity (British white) } \\
\qquad N=1912\end{array}$ & $98(43 \%)$ & $803(42 \%)$ & $901(42 \%)$ \\
\hline $\begin{array}{l}\text { Computed tomography scan (n \% yes) } \\
\qquad \mathrm{N}=2112\end{array}$ & $30(13 \%)$ & $311(16 \%)$ & $341(16 \%)$ \\
\hline $\begin{array}{l}\text { Intubation ( } \mathrm{n} \% \text { yes) } \\
\qquad \mathrm{N}=2115\end{array}$ & $8(4 \%)$ & $76(4 \%)$ & $84(4 \%)$ \\
\hline $\begin{array}{l}\text { Median GCS score (range) } \\
N=2081\end{array}$ & $15(12)$ & $15(14)$ & $15(14)$ \\
\hline \multicolumn{4}{|l|}{ Diagnosis (ICD code) $N=2137$} \\
\hline External causes of morbidity (V01-Y98) & $39(17 \%)$ & $235(12 \%)$ & $274(13 \%)$ \\
\hline Diseases of the circulatory system (100-199) & $84(37 \%)$ & $776(41 \%)$ & $860(40 \%)$ \\
\hline Diseases of the respiratory system (J00-J99) & $25(11 \%)$ & $287(15 \%)$ & $312(15 \%)$ \\
\hline Diseases of the nervous system (G00-G99) & $15(6 \%)$ & $101(5 \%)$ & $116(5 \%)$ \\
\hline Mental and behavioural disorders (F00-F99) & $11(5 \%)$ & $90(5 \%)$ & $101(5 \%)$ \\
\hline Diseases of the digestive system (K00-K93) & $10(4 \%)$ & $115(6 \%)$ & $125(6 \%)$ \\
\hline Other & $45(20 \%)$ & $304(16 \%)$ & $349(16 \%)$ \\
\hline
\end{tabular}

${ }^{*} p<0.01$. BAC, blood alcohol concentration; GCS, Glasgow coma scale; ICD, International Classification of Diseases.

GmbH, Passau, Germany) on an Olympus AU2700 analyser (Olympus Optical Co Ltd). BAC are reported in $\mathrm{mg} / 100 \mathrm{ml}$; less or equal to 10 approximated to $\mathrm{BAC}$ negative and more than 10 was BAC positive.

Electronic and written $\mathrm{ED}$ and hospital records were subsequently examined by researchers masked to the BAC results. For those patients transferred to seven other hospitals, records were similarly examined for length of stay and outcome. In addition to basic demographic information, the time, date and reason for presentation to the RR were recorded, together with Glasgow coma scale (GCS) scores, information about immediate management (intubation/whether they received a computed tomography scan), final primary diagnosis (using International Classification of Diseases (ICD) version 10 codes $^{13}$ for consistent standardisation), length of stay, return visit(s) to the ED and mortality over the following 6 months.

Trauma severity was rated by an independent team from the UK trauma audit and research network, commissioned for this study. All trauma cases were assessed using the injury severity score. This method uses the abbreviated injury scale scores based on the severity of the injury, which is applied to the regions of the body ${ }^{14}$ The minimum score is 0 , whereas the maximum score is 75 . An injury severity scale score of less than 12 was used to classify minor trauma.

Each blood sample was assigned an identifying number to log the BAC results into an Excel spreadsheet that was not accessible by the treatment team. All data were then entered onto a database and analysed using SPSS (version 14.0). Univariate and multivariate tests were used to compare the characteristics and outcomes of those with positive and negative BAC. The impact of a positive BAC on management and outcomes was examined using binary logistic regression. For rates and proportions, 95\% CI were calculated.

\section{RESULTS}

There were 2326 direct patient presentations to the RR during the course of the study, 22 August 2005 to 31 August 2006. Of these, 2137 patients were eligible to participate, but 229 of these were not tested (fig 1). The characteristics of those who were tested versus those who were not tested are presented in table 1. There were no differences between the groups except that men were less likely to have BAC tested.

Of the 1908 with BAC results, 1617 had BAC scores of less than $11 \mathrm{mg} / 100 \mathrm{ml}$ (BAC negative). The remaining 291 (15\%) had a positive $\mathrm{BAC}$, ranging from 11 to $574 \mathrm{mg} / 100 \mathrm{ml}$. The characteristics of those BAC negative are compared with those with a positive test in table 2 . The top five RR presentations associated with a positive BAC were collapse due to alcohol/drugs, self-harm, trauma, gastrointestinal bleeding and non-cardiac chest pain. An independent samples $t$ test showed that those who were BAC positive had a mean age of 45.4 years compared with 58.9 years among those who were BAC negative (difference in means 4.99; $95 \%$ CI 10.97 to 15.96 ). Using binary logistic regression, men were found to be more likely to be positive than women (odds ratio (OR) $2.27,95 \%$ CI 1.17 to 3.03 ).

BAC were divided into ICD-10 diagnoses (table 2) and Y90.18 (BAC) codes (fig 2). A total of 110 (38\% of 291 positives) had a BAC of over $240 \mathrm{mg} / 100 \mathrm{ml}$ (Y90.8), of which 62 were over $300 \mathrm{mg} / 100 \mathrm{ml}(21 \%), 18$ were over $400 \mathrm{mg} / 100 \mathrm{ml}(6 \%)$ and four were over $500 \mathrm{mg} / 100 \mathrm{ml}(1 \%)$.

In our study, collapse due to alcohol misuse was classified under mental and behavioural disorders, F10 (F10.0 acute intoxication: with 10.1 harmful use, 10.2 dependent use, 10.3 withdrawal state). ${ }^{13}$ Of the 45 such patients, four (9\%) had a BAC of less than $11 \mathrm{mg} / 100 \mathrm{ml}$. Of the 22 who were classified as primarily drug abuse (F11-F19), nine (41\%) had a positive $\mathrm{BAC}$, the association between the two being well recognised. Alcohol misuse may also be coded either Y90 (fig 2), with subcodes defined by a series of nine BAC levels, or $\mathrm{Y} 91$ by grades of clinical assessment in the absence of BAC.

Using binary logistic regression, the 196 incidents involving trauma (accidents, assault or self-harm-comprising only 10\% of 1908) were found to be more likely to be BAC positive than other presentations (OR 3.52; 95\% CI 2.54 to 4.0), apart from collapse due to alcohol/drugs. Those with a high trauma score were no more likely to be BAC positive than those with a low 
Table 2 Characteristics of 1908 episodes with a positive and negative BAC

\begin{tabular}{|c|c|c|}
\hline & $\begin{array}{l}\text { Alcohol } \\
<10 \mathrm{mg} / 100 \mathrm{ml}\end{array}$ & $\begin{array}{l}\text { Alcohol } \\
>10 \mathrm{mg} / 100 \mathrm{ml}\end{array}$ \\
\hline & $\begin{array}{l}N=1617 \\
(85 \%)\end{array}$ & $\begin{array}{l}N=291 \\
(15 \%)\end{array}$ \\
\hline Gender (male)* & $948(59 \%)$ & $251(86 \%)$ \\
\hline Mean age $(S D)^{*}$ & $58.85(20.47)$ & $45.38(17.63)$ \\
\hline Ethnicity (British white) & $654(40 \%)$ & $149(51 \%)$ \\
\hline Reason (ICD code) & $\mathrm{N}$ and $\%$ for each code & \\
\hline \multicolumn{3}{|l|}{ External causes of morbidity (V01-Y98) } \\
\hline Road traffic accident (V01-99) & $43(81 \%)$ & $10(19 \%)$ \\
\hline Other accidents (W00-X59) & $53(62 \%)$ & $36(38 \%)$ \\
\hline Assault (X85-Y09) & $28(57 \%)$ & $21(43 \%)$ \\
\hline Self-harm (X60-X84) & $23(51 \%)$ & $22(49 \%)$ \\
\hline \multicolumn{3}{|l|}{ Diseases of the circulatory system (100-199) } \\
\hline Ischaemic heart disease (I20-I25) & $358(90 \%)$ & $40(10 \%)$ \\
\hline Arrhythmic/conduct disorders (144-145) & $110(95 \%)$ & $6(5 \%)$ \\
\hline Congestive cardiac failure $(150)$ & $69(93 \%)$ & $5(7 \%)$ \\
\hline Myocardial infarction (I21) & $46(94 \%)$ & $3(6 \%)$ \\
\hline Cerebral infarct/haemorrhage (160-164) & $67(92 \%)$ & $6(8 \%)$ \\
\hline Other & $60(91 \%)$ & $6(9 \%)$ \\
\hline \multicolumn{3}{|l|}{ Diseases of the respiratory system (J00-J99) } \\
\hline Chronic obstructive pulmonary disease (J43-J44) & $70(96 \%)$ & $3(4 \%)$ \\
\hline Asthma (J45) & $51(90 \%)$ & $6(10 \%)$ \\
\hline Bronchitis/emphysema (J40-J42) & $64(97 \%)$ & $2(3 \%)$ \\
\hline Other & $85(94 \%)$ & $6(6 \%)$ \\
\hline \multicolumn{3}{|l|}{ Diseases of the nervous system (G00-G99) } \\
\hline Paroxysmal disorders (epilepsy) (G40-G47) & $74(88 \%)$ & $10(12 \%)$ \\
\hline Other & $16(94 \%)$ & $1(6 \%)$ \\
\hline \multicolumn{3}{|l|}{ Mental and behavioural disorders (F00-F99) } \\
\hline Alcohol misuse $(F 10)$ & $4(9 \%)$ & $41(91 \%)$ \\
\hline Drug misuse (F11-F19) & $13(59 \%)$ & $9(41 \%)$ \\
\hline Acute confusional state (F04) & $5(100 \%)$ & $0(0 \%)$ \\
\hline Other & $14(78 \%)$ & $4(22 \%)$ \\
\hline \multicolumn{3}{|l|}{ Diseases of the digestive system ( $\mathrm{KOO}-\mathrm{K} 93$ ) } \\
\hline Gastrointestinal bleed (K92.2) & $34(77 \%)$ & $10(23 \%)$ \\
\hline Other & $65(92 \%)$ & $6(8 \%)$ \\
\hline \multicolumn{3}{|l|}{ Other } \\
\hline Acute infection/sepsis (A00-B99) & $35(88 \%)$ & $5(12 \%)$ \\
\hline Non-cardiac chest pain (R07) & $45(76 \%)$ & $14(24 \%)$ \\
\hline Syncope (R53) & $32(97 \%)$ & $1(3 \%)$ \\
\hline Obstetric/gynaecological (000-099) & $9(90 \%)$ & $1(10 \%)$ \\
\hline Anaphylaxis (T78.2) & $20(83 \%)$ & $4(17 \%)$ \\
\hline Miscellaneous & $124(90 \%)$ & $13(10 \%)$ \\
\hline
\end{tabular}

${ }^{*} \mathrm{p}<0$.01. BAC, blood alcohol concentration; ICD, International Classification of Diseases.

score: the mean BAC in those with a score below 12 was 68.1 compared with 76.6 in those with a trauma score above 12 (difference in means 8.5 ; $95 \%$ CI 0.46 to 2.45 ). Nearly half (46\%) of patients with head and neck trauma were BAC positive, which demonstrated a trend towards significance (OR 1.91; 95\% CI 0.97 to 3.82 ).

Running a binary logistic regression, those BAC positive were found to be more likely to have been intubated while in the resuscitation room (OR 1.80; 95\% CI 0.99 to 3.27), even after taking into account the confounding effect of their presenting condition. However, they were not more likely to have been sent for a computed tomography scan (OR 1.17; 95\% CI 0.79 to 1.73). Of the 1858 (97.4\%) who had a GCS score documented in their notes, the median score was lower when BAC positive $(z=-5.26, p<0.001)$. A total of $1412(74 \%)$ of the 1908 presentations to the RR resulted in admission to hospital.

Regarding 6-month outcomes, of the 1753 patients (155 were return visits) who presented to our $R R$ and had a BAC measured, 494 (28\%) subsequently visited our ED during the following 6 months. It is notable that 219 (12\%) died within 6 months, according to computer data held by our hospital (which records later patient death for those previously attending our ED). When taking into account the confounding effects of age and presenting condition, a positive BAC was associated with an increased likelihood of a return visit to the ED within 6 months (OR 1.46; 95\% CI 1.03 to 2.05). The likelihood of admission to a ward on first attendance to the RR (OR 1.12; 95\% CI 0.80 to 1.58) or death within 6 months (OR 1.21; 95\% CI 0.66 to 2.21) were also higher among those with a positive BAC, but these were not statistically significant.

\section{DISCUSSION}

This is the first such report assessing BAC taken over one year specifically from RR patients. We show that more than one in seven people treated in our RR over a 12-month period have 
3214 direct presentations to the resuscitation room

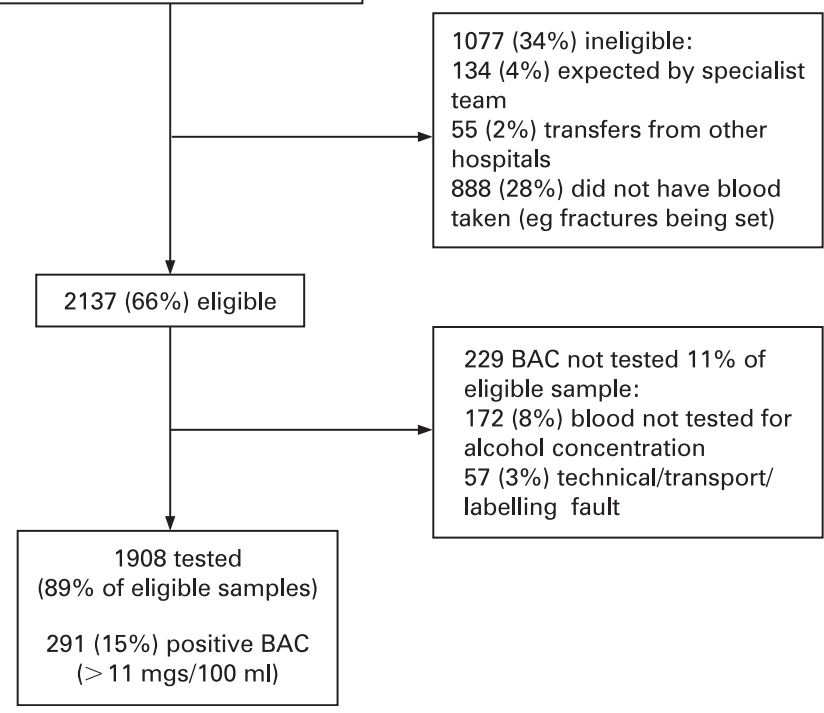

Figure 1 Flow diagram. BAC, blood alcohol concentration.

consumed alcohol before their presentation. Half of all those treated following self-harm or assault will have a positive BAC. Injury to the head and neck (S00-S19) was associated with a positive BAC, being related to assault. ${ }^{15}$ BAC should be requested when clinicians judge it would be helpful, but our study highlights the presentations that are more likely to be associated with an elevated BAC, for which we recommend a $\mathrm{BAC}$ be included in the initial tests requested. We also recommend that positive $\mathrm{BAC}$ results be followed up by brief advice/brief intervention when the patient is no longer in the RR. ${ }^{1}$ Although a positive BAC can help a clinician clarify the role that alcohol use may have played in the patient's presentation, a positive BAC alone is not a sound basis for making judgements about the cause of the patient's presenting complaint. A negative, or low, BAC is a definitive exclusion for reduced consciousness.

Patients presenting with trauma showed no relationship between severity, as defined by trauma scoring using the TRISS methodology ${ }^{14}$ and BAC level. Findings from different studies examining the relationship between alcohol and injury severity continue to be conflicting. ${ }^{16}$ Of the 196 trauma cases reported here, only 27 (14\%) had a trauma score of 12 or more.

Requesting a BAC should be according to clinical judgement as for any investigation, ${ }^{1}$ but for the five presentations of collapse due to alcohol/drugs, self-harm, trauma, gastrointestinal bleeding and non-cardiac chest pain, we suggest BAC should be part of the initial set of blood tests obtained from the patients in the RR. We have demonstrated a higher rate of return visits and a trend towards higher mortality in the 6 months following a BAC-positive patient's treatment in the RR. Davidson et $a l^{17}$ have previously confirmed poor outcomes for intoxicated ED patients (BAC >100 mg/100 ml) over a subsequent 5 -year period, but these were not specifically from the RR. ${ }^{17}$

All ED systems are a reflection of healthcare practice and medical culture; BAC is a universal standard, laboratory facilities permitting. ${ }^{18}$ On its own, BAC is not as effective for detection as a questionnaire; ${ }^{19}$ however, it is a simple method of detection in the RR that can lead to subsequent brief advice/ intervention depending on the $\mathrm{ED}$ environment, time, training

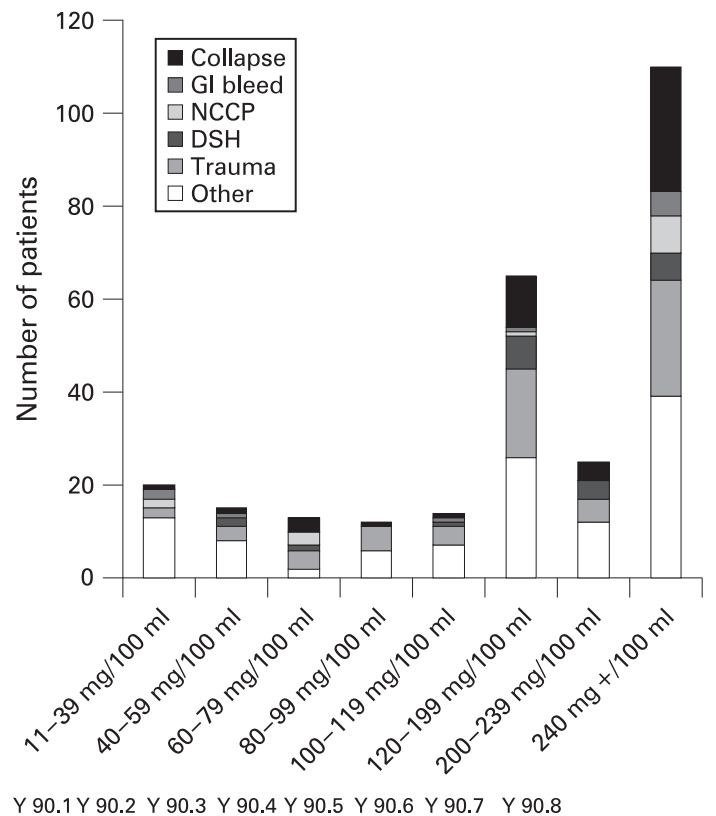

Figure 2 Blood alcohol concentration: prevalence by $Y 90$ coding. DSH, deliberate self-harm; Gl, gastrointestinal; NCCP, non-cardiac chest pain.

and availability. ${ }^{20}$ This approach has now been recognised in national guidance to level 1 trauma centres in the USA, stating that all patients must be screened for alcohol misuse and provided brief advice/intervention. ${ }^{21}$ Internationally, ED may need economic incentives to encourage similar service provision to reduce re-visits. ${ }^{22}$ A recent survey of 191 ED in England revealed that almost half do not have systems in place to allow BAC testing to occur. ${ }^{18}$

Our study is confined to RR patients initially attending a single urban ED in a metropolitan area. The extent to which these data can be generalised to other ED is unclear. Although drinking patterns in the area served by our ED are not atypical of those in other densely populated areas in the UK, ${ }^{23}$ levels of alcohol use among patients attending other ED RR are not known.

In our study BAC results were unavailable to the medical staff; BAC were not previously routinely requested from our $\mathrm{RR}$, nor was BAC testing available on site. The results of testing remained confidential, follow-up (ie, brief advice) not yet organised. ${ }^{1}$ Those involved in collecting outcome data on investigations and return visits to the $\mathrm{ED}$ were also unaware of results in order to minimise the likelihood of bias. We therefore did not study the influences that BAC results may have had on patient management. ${ }^{10}$

As alcohol is metabolised at approximately $15 \mathrm{mg} / 100 \mathrm{ml}$ per hour, ${ }^{24}$ the delay between consuming alcohol and BAC being measured on arrival at the RR means that our data are likely to underestimate levels of misuse. Patients have diminished ability to recall past consumption as BAC increases, so that self-report is increasingly unreliable as consumption increases. ${ }^{25}$ Chronic heavy drinkers have developed both pharmacological and psychological tolerance, whereas the non-dependent binge drinker is more vulnerable. ${ }^{2}$

It is simple to add BAC to an initial set of RR blood investigations, a fluoride-oxalate bottle being used for both sugar and BAC estimations. BAC provides an important and inexpensive means of identifying the possible role, or not, of alcohol in a patient's presenting complaint and furthermore, 
may act as "an alert" to counter possible clinical inertia of the treating doctor. ${ }^{26}$ The health consequences of alcohol misuse for those treated in the RR are potentially even greater than those treated elsewhere in the ED. Such patients, providing they survive, may therefore be even more likely to contemplate change, ${ }^{27}$ and be keen to avoid future risky behaviour and untoward incidents. BAC should routinely be considered for all $\mathrm{RR}$ patients with collapse from alcohol/drugs, trauma and intentional self-harm as well as for gastrointestinal bleeding and non-cardiac chest pain.

Acknowledgements: The authors gratefully acknowledge the work and cooperation of all the ED staff, especially the nursing staff and senior house officers of teams 39 and 40, for the initial collection of samples in the RR; also Dr Patricia Ward, lead clinician. In addition, they wish to thank St Mary's chemical pathology laboratory staff for their work in analysing samples, particularly Dr Bill Richmond and Stephen Snewin. They are very grateful to Maralyn Woodford and Laura White from the trauma audit and research network for help with the trauma scoring. ${ }^{14}$ to Dr Alex Paton and Dr lan Maconochie for their editorial advice and especially to Margaret Bennett, health records service manager.

Funding: The study was funded by St Mary's Paddington Charitable Trust, from a grant resulting from a donation of $£ 125000$ from Railtrack plc out of appreciation of St Mary's response to the Paddington rail crash of 5 October 1999. The funder (and the donor) played no part in the conduct or reporting of the study.

Competing interests: None.

Ethics approval: The local research ethics committee approved this study as an audit, therefore informed consent was waived (no EC2143 dated 31 March 2005).

Contributors: RT, MJC, EC, PH, AB and NB designed the study protocol. EC, TP, PG, $H M, A J S$ and $A B$ collected study data. PH led the laboratory work. All authors helped analyse data, interpret findings and write the paper. $\mathrm{RT}$ is the guarantor for the paper and accepts full responsibility for the conduct of the study, had access to the data and controlled the decision to publish.

\section{REFERENCES}

1. Csipke $\mathbf{E}$, Touquet $R$, Patel $T$, et al. Use of blood alcohol concentration in resuscitation room patients. Emerg Med J 2007;24:535-8.

2. Cherpitel CJ. Alcohol and injuries: a review of international emergency room studies since 1995. Drug Alcohol Rev 2007;26:201-14.

3. Ahmed M, Mackway-Jones K. Is emergency department based brief intervention worthwhile in adults presenting with alcohol related events? Emerg Med J 2007:24:785-6.

4. Crawford MJ, Patton R, Touquet R, et al. Screening and referral for brief intervention of alcohol-misusing patients in an emergency department: a pragmatic randomized controlled trial. Lancet 2004;364:1334-9.
5. Patton R, Hilton C, Crawford MJ, et al. The Paddington alcohol test: a short report. Alcohol Alcohol 2004;39:266-8.

6. Cherpitel C, Bond J, Ye Y, et al. Clinical assessment compared with breathalyser readings in the emergency room: concordance of ICD-10 Y90 and Y91 codes. Emerg Med J 2005;22:689-95.

7. Urso T, Gavaler JS, Van Thiel DH. Blood ethanol levels in 'sober' alcohol users seen in an emergency room. Life Sci 1981;28:1053-6.

8. Savola 0, Niemela 0, Hillbom M. Blood alcohol is the best indicator of hazardous alcohol drinking in young adults and working-age patients with trauma. Alcohol Alcohol 2004;39:340-5.

9. Peppiatt R, Evans R, Jordan P. Blood alcohol concentrations of patients attending an accident and emergency department. Resuscitation 1978;6:37-43.

10. Jurkovich GJ, Rivara FP, Gurney JG, et al. Effects of alcohol intoxication on the initial assessment of trauma patients. Ann Emerg Med 1992;21:704-8.

11. Gentilello LM. Confronting the obstacles to screening and interventions for alcohol problems in trauma centers. J Trauma 2005;59:S137-43.

12. Manchester Triage Group. Emergency triage. 2nd ed. Oxford, UK: BMJ Books, Blackwell Publishing, 2006.

13. World Health Organization. ICD-10: International Statistical Classification of Diseases and Related Health Problems, 10th revised ed. Geneva: World Health Organization, 1992.

14. Woodford M. Scoring systems for trauma. In: Driscoll P, Skinner D, Earlam R, eds. $A B C$ of major trauma, 3rd ed. London: BMJ Books, Blackwell Publishing, 2005

15. Smith AJ, Hodgson RJ, Bridgeman $\mathrm{K}$, et al. A randomized trial of a brief intervention after alcohol-related facial injury. Addiction 2003;98:43-52.

16. Li G, Keyl PM, Smith GS, et al. Alcohol and injury severity: reappraisal of the continuing controversy. J Trauma 1997;42:562-9.

17. Davidson P, Koziol-McLain J, Harrison L, et al. Intoxicated ED patients: a 5-year follow-up of morbidity and mortality. Ann Emerg Med 1997;30:593-7.

18. Patton R, Strang J, Birtles C, et al. Alcohol: a missed opportunity. A survey of all AEDs in England. Emerg Med J 2007;24:529-31.

19. Cherpitel CJ, Soghikian K, Hurley LB. Alcohol-related health services use and identification of patients in the ED. Ann Emerg Med 1996;28:418-23.

20. Hungerford DW, Pollock DA. Emergency department services for patients with alcohol problems: research directions. Acad Emerg Med 2003;10:79-84.

21. American College of Surgeons, Committee on Trauma. Resources for optimal care of the injured patient. Chicago, IL: American College of Surgeons, 2006.

22. Cherpitel CJ. Alcohol-related injury and the emergency department: research and policy questions for the next decade. Addiction 2006;101:1225-7.

23. London Health Observatory. Lifestyle and behaviour: alcohol use in London. London: London Health Observatory, 2003.

24. Paton A. Alcohol in the body. In: Paton A, Touquet R, eds. $A B C$ of alcohol, 4th ed London: BMJ Books, Blackwell Publishing, 2005.

25. Moore S, Shepherd J, Perham N, et al. The prevalence of alcohol intoxication in the night-time economy. Alcohol Alcohol 2007:42:629-34.

26. Huntley JS, Patton, Touquet R. Attitudes towards alcohol of emergency department doctors trained in the detection of alcohol misuse. Ann $R$ Coll Surg Engl 2004;86:329-33.

27. Rollnick S, Butler CC, McCambridge J, et al. Consultations about changing behaviour. BMJ 2005;331:961-3. 